\title{
Sleep Leads to Changes in the Emotional Memory Trace: Evidence from fMRI
}

\author{
Jessica D. Payne ${ }^{1}$ and Elizabeth A. Kensinger ${ }^{2,3}$
}

\begin{abstract}
After information is encoded into memory, it undergoes an off-line period of consolidation that may occur optimally during sleep. The consolidation process not only solidifies memories but also changes them in ways that render them more useful and adaptive. Here, we provide evidence for a shift in the neural structures used to retrieve emotional memories after a night of sleep. Although the hippocampus was activated during successful retrieval of negative objects regardless of whether participants slept during a delay, sleep led to a shift from engage-
\end{abstract}

\section{INTRODUCTION}

Memory consolidation is a time-dependent, off-line collection of neurobiological processes that stabilize memories against interference and decay and allow them to persist over time (Dudai, 2004; McGaugh, 2000). The neurobiological milieu of the brain during sleep is thought to provide ideal conditions for such stabilization to occur (Payne, Ellenbogen, Walker, \& Stickgold, 2008; Marshall \& Born, 2007; Rasch \& Born, 2007), and growing evidence, spanning numerous levels of analysis, demonstrates that sleep plays an important role in the consolidation of both procedural and declarative memories (Stickgold, 2005; Wilson, 2002).

Successful consolidation is typically observed quantitatively as enhancement of skills after sleep in the case of procedural learning (Walker \& Stickgold, 2006) and as resistance to deterioration after sleep in the case of episodic memory (Payne et al., 2009; Payne, Stickgold, Swanberg, \& Kensinger, 2008; Gais, Lucas, \& Born, 2006; Jenkins \& Dallenbach, 1924). As these examples suggest, the notion of consolidation implies that memories are solidified in a veridical manner, true to their form at initial encoding, and sleep helps enhance or maintain specific skills or information. Yet substantial evidence demonstrates that declarative memories can also change with the passage of time (Roediger \& McDermott, 2000; McDermott, 1996; Schacter, 1996; Bartlett, 1932), suggesting that the process of consolidation does not always yield exact representa-

${ }^{1}$ University of Notre Dame, ${ }^{2}$ Boston College, ${ }^{3}$ Athinoula A. Martinos Center for Biomedical Imaging, Charlestown, MA ment of a diffuse memory retrieval network-including widespread activity in the lateral prefrontal and parietal corticesto a more refined network of regions-including the amygdala and ventromedial pFC. Effective connectivity analyses revealed stronger connections among limbic regions after sleep versus wake. Although circadian effects may have contributed to these findings, our data strongly suggest that a night of sleep is sufficient to evoke qualitative changes in the emotional memory retrieval network.

tions of experience. On the face of it, this idea may seem maladaptive, yet such flexibility in memory representation allows the emergence of key cognitive abilities, such as generalization and inference (Ellenbogen, $\mathrm{Hu}$, Payne, Titone, \& Walker, 2007; Fenn, Nusbaum, \& Margoliash, 2003), and adaptive functions such as the selective preservation of useful information extracted from a barrage of incoming stimulation and experience (Payne et al., 2009).

Consistent with these ideas, growing behavioral evidence suggests that sleep does more than simply consolidate memories in veridical form; it also transforms them in ways that render memories less accurate in some respects but perhaps more useful and adaptive in the long run. For example, sleep leads to flexible restructuring of memory traces so that insights and abstractions can be made (Gomez, Bootzin, \& Nadel, 2006; Wagner, Gais, Haider, Verleger, \& Born, 2004), inferences can be drawn (Ellenbogen et al., 2007), integration can occur (Payne et al., 2009; Dumay \& Gaskell, 2007), and importantly for our purposes here, emotionally salient aspects of information can be preferentially remembered over less salient, neutral aspects (Payne, Stickgold, et al., 2008).

Importantly, each of these behavioral studies reveals qualitative rather than quantitative changes in memory as a function of nocturnal sleep compared with daytime wakefulness, and many of these changes emerge over a relatively brief 12-hour delay containing sleep. Moreover, in each study, sleep confers a flexibility to memory that may be at times more advantageous than a literal representation of experience (Payne et al., 2009). Although these behavioral studies suggest that sleep leads to a shift in the way memories are stored and retrieved, neuroimaging 
methods provide a direct test of whether sleep confers a genuine change in the neural systems supporting memory. An essential question, then, is whether the behavioral changes observed in memory after sleep are a reflection of changes in mnemonic processing at the level of the brain.

To address this question, we examined memory performance after a 12-hour delay containing either nocturnal sleep or daytime wakefulness. This design allowed us to examine whether a single night of sleep was sufficient to provoke changes in underlying memory retrieval networks or rather increase activity within the identical memory network recruited after a period of wakefulness. We chose to investigate this question in the realm of emotional memory because numerous behavioral studies have demonstrated that nocturnal sleep benefits the consolidation of emotional relative to neutral memories compared with daytime wakefulness (Payne, Stickgold, et al., 2008; Hu, Stylos-Allan, \& Walker, 2006; Wagner, Hallschmid, Rasch, \& Born, 2006; Wagner, Gais, \& Born, 2001). For example, we recently examined how different components of negative emotional memories change across periods of nocturnal sleep versus daytime wakefulness, demonstrating that sleep selectively preserves memory for negative objects within emotional scenes at the expense of neutral background details (Payne, Stickgold, et al., 2008). Although daytime wakefulness led to forgetting of emotional scenes in their entirety, with both objects and backgrounds decaying at similar rates, sleep led to a selective preservation of negative objects, but not the backgrounds, suggesting that the two components undergo differential processing during sleep.

This finding suggests that negative scene memories develop differentially across time delays containing nocturnal sleep versus daytime wakefulness and that rather than preserving intact representation of scenes, sleep effectively "unbinds" scenes to consolidate only their most emotionally salient and perhaps adaptive emotional element. We have shown that this effect is further intensified after a 24-hour delay, but only when sleep directly follows encoding of the emotional scenes (Payne et al., submitted). Moreover, we have demonstrated that emotional objects are better remembered after a 90-min nap than after two different wake control conditions that strictly controlled for circadian and interference influences (Payne et al., submitted; for complete study description, see the Alternative explanations for the present findings section). Collectively, these results suggest that sleep-mediated consolidation processes solidify the negative emotional aspects of an experience into a durable memory while allowing the less emotional aspects to decay.

In the present study, we used the same paradigm to examine the neural regions associated with the retrieval of emotional object memories after off-line periods containing either nocturnal sleep or daytime wakefulness, predicting that different neural systems would be used to retrieve these memories after time spent in these distinct brain states. Specifically, we hypothesized that (1) activity in the amygdala, medial-temporal lobe memory system (particularly the hippocampus), and ventromedial pFC (VMPFC) would be more active during retrieval of negative objects after sleep than after wakefulness and (2) retrieval of negative objects would be associated with increased effective connectivity among these areas after sleep relative to wakefulness, reflecting the outcome of superior emotional memory processing during sleep-dependent consolidation.

\section{METHODS}

\section{Participants}

Participants were 42 native English speakers from Boston College and Harvard University (ranging from 18 to 29 years of age, with a mean age of 22.1 years), with normal or corrected-to-normal vision. They were screened for neurological, psychiatric, and sleep disorders and for medications affecting the CNS or sleep architecture. Participants were randomly assigned to a wake delay (21 participants13 women) or a sleep delay (21 participants-11 women) condition. Individuals comprising the sleep-delay and wakedelay groups did not differ in age, education, and scores on the Morningness-Eveningness Questionnaire (Horne \& Ostberg, 1976), Beck Depression Inventory (Beck \& Beamesderfer, 1974) or the Beck Anxiety Inventory (Beck, Epstein, Brown, \& Steer, 1988) (all $p$ s > .15).

Participants in the wake-delay group encoded images between 7:00 and 9:00 a.m. and retrieved them from memory while undergoing an fMRI scan 12 hours later, between 7:00 and 9:00 p.m. Napping was not permitted between sessions, and participants were excluded from analyses if debriefing indicated that they had napped during the delay. Participants in the sleep-delay group encoded the images between 8:00 and 10:00 p.m. and retrieved them 12 hours later in the scanner, after at least 6.5 hours of sleep confirmed by questionnaire. Sleep amount was statistically equivalent in the wake and sleep groups $(t<$ $1.8, p>.25)$. Data from three participants were excluded (two male sleep-delay participants due to excessive head movement and one female wake-delay participant for napping during the delay interval), resulting in a total of 39 participants used in the below analyses.

\section{Materials}

Two versions of 120 scenes were created by placing either a negative arousing or a neutral object on a plausible neutral background (e.g., by placing either a chipmunk or a snake on a forest background). Stimuli were taken from those used in Payne, Stickgold, et al. (2008) and were supplemented with images from Waring and Kensinger (2009). Objects and backgrounds were previously rated for valence and arousal using 7-point scales. All negative objects were given arousal ratings of 5-7 (with high scores signifying an exciting or arousing image) and valence ratings 
lower than 3 (with low scores signifying a negative image). All neutral items (objects and backgrounds) were rated as nonarousing (arousal values lower than 4) and neutral (valence ratings between 3 and 5; Waring \& Kensinger, 2009; Kensinger, Garoff-Eaton, \& Schacter, 2007).

\section{Behavioral Procedure}

During the encoding session, participants studied a set of 80 scenes ( 40 with a neutral object and 40 with a negative object, all on neutral backgrounds) for 5 sec each and then indicated whether they would choose to approach or move away from the scene if they encountered it in real life (to ensure deep encoding). After the delay period, participants performed an unexpected recognition task while undergoing an fMRI scan. Debriefing confirmed that no participant expected their memory would be assessed; most participants believed that they would be making similar decisions during the fMRI scan to those they had made in the earlier study session. Participants viewed objects and backgrounds, presented separately and one at a time, and indicated whether each item was "old" (included in a previously studied scene) or "new" (not previously studied). Items included on the recognition test were 80 old objects (40 neutral, 40 negative), 80 old backgrounds (40 studied with a neutral object and 40 with a negative object), 80 new objects (40 negative, 40 neutral), and 40 new backgrounds (by definition, all neutral). These item types were pseudorandomly intermixed with one another, and ISIs were jittered from between 2 and 14 sec to optimize the ability to isolate the hemodynamic response associated with each item's presentation (Dale, 1999). A short practice was performed before entering the scanner to assure that all participants understood the instructions and the timing of the recognition task.

\section{fMRI Image Acquisition and Preprocessing}

Data were acquired on a 1.5-T Siemens whole-body Avanto MRI scanner (Erlangen, Germany) using a standard birdcage head coil. The stimuli were projected from a Macintosh iBook G4 to a Sharp200 color LCD projector through a collimating lens that projected onto a screen mounted in the magnet bore. Participants viewed the screen through mirrors located on the head coil.

Anatomic data were acquired with a multiplanar rapidly acquired gradient-echo (MP-RAGE) sequence (repetition time $=2730 \mathrm{msec}$, echo time $=3.31 \mathrm{msec}$, flip angle $=40^{\circ}$, field of view $=256 \times 256 \mathrm{~mm}$, acquisition matrix $256 \times 256$, slice thickness $=128$, slice thickness $=$ $1.33 \mathrm{~mm}$, no gap, $1 \times 1 \times 1.33 \mathrm{~mm}$ resolution). Coplanar and high-resolution T1-weighted localizer images were acquired. In addition, a T1-weighted inversion recovery echo-planar image was acquired for auto alignment.

Functional images were acquired via a $\mathrm{T} 2 *$-weighted EPI sequence sensitive to the BOLD signal, with a repetition time of $2000 \mathrm{msec}$, an echo time of $40 \mathrm{msec}$, and a flip angle of $90^{\circ}$. Twenty-six interleaved axial-oblique slices (parallel to the line between the anterior and the posterior commissures) were collected in a $3.125 \times 3.125 \times$ 3.72-mm matrix (with a 3.12 thickness and a 0.6-mm skip between slices).

Preprocessing and data analysis were completed using SPM2 (Statistical Parametric Mapping; Wellcome Department of Cognitive Neurology, London, UK). Slice time correction was completed, and motion correction was run, using a six-parameter, rigid-body transformation algorithm by SPM2. The images were normalized to the Montreal Neurological Institute (MNI) template. The resultant voxel size was $3 \times 3 \times 3 \mathrm{~mm}$, and spatial smoothing was completed at a 7.6-mm isotropic Gaussian kernel.

\section{Event-related fMRI Data Analysis}

Analyses focus specifically on responses to objects that had been included in studied scenes, examining the influence of object valence (negative, neutral) and memory performance (remembered, forgotten) on neural activity in the sleep and wake group. Thus, analyses focus on responses to four different object types: remembered negative objects, forgotten negative objects, remembered neutral objects, and forgotten neutral objects. For each participant, on a voxel-by-voxel basis, these event types were modeled through convolution with a canonical hemodynamic response function. This yielded beta-weights for each voxel. For statistical contrasts conducted within a single group (e.g., comparing remembered with forgotten negative items within the sleep-delay group), voxels were considered active when the difference between beta-weights was statistically positive as determined by a one-tailed paired $t$ test with variance estimated using random-effect analysis. For statistical contrasts conducted to compare the wake-delay and the sleep-delay groups, voxels were considered active when the difference between beta-weights was statistically positive as determined by a one-tailed twosample $t$ test. Unless otherwise specified, only regions that consist of at least five contiguous voxels, with peak activity at $p<.001$, are reported in the results.

Conjunction analyses, using the masking function in SPM2, were used to reveal the regions that were active for specific contrasts in both the sleep-delay and the wake-delay groups. The individual contrasts included in the contrast analysis were analyzed at a threshold of $p<.01$ [such that the conjoint probability of the conjunction analysis, using Fisher's estimate (Lazar, Luna, Sweeney, \& Eddy, 2002; Fisher, 1950), was $p<.001]$. Masking procedures were used to reveal regions that were active in one group (at $p<.001$ ) but not in the other group (even when the threshold was lowered to $p<.10$ ).

To depict the pattern of activity within ROIs, 8-mm spheres were created around regions identified within contrast analyses, and signal change within these spheres was estimated using the MarsBar toolbox implemented within SPM2 (Brett, Anton, Valabregue, \& Poline, 2002). 
For each region and for each object type, the average of the signal change reached between 4 and 6 sec poststimulus onset was measured for each trial type, and these values were used to calculate the magnitude of the accurate retrieval response for negative and for neutral objects (e.g., activity to forgotten negative objects was subtracted from activity to remembered negative objects). These memory effects (changes in percent signal change) are depicted in the bar graphs accompanying Figures 1 and 2 and are presented for illustrative purposes only. Tables report voxel coordinates in both MNI and Talairach (Talairach \& Tournoux, 1988) coordinates at the peak voxel in each cluster. All activations are presented in neurological coordinates and are displayed on canonical images provided within SPM2.

To examine the connectivity among a priori ROIs in the amygdala, hippocampus, VMPFC, and fusiform gyrus, structural equation modeling (SEM) was carried out using Lisrel software (Joreskog \& Sorbom, 1993). So that the ROIs would be defined in a fashion that was unbiased with regard to emotion or memory accuracy, each ROI was defined functionally from a contrast that compared all item retrieval trials with the fixation baseline; the MNI coordinates of the peak voxel within each ROI were as follows: amygdala $(-16,0,-22)$, hippocampus $(-19,-14,-21)$, VMPFC $(4,56,-8)$, and fusiform gyrus $(-30,-28,-20)$. We had a priori hypotheses (as outlined in the introduction) for the effect of sleep on amygdala, hippocampal, and VMPFC connectivity. Although we did not have any specific hypotheses about fusiform connectivity, we felt it was important to include this region in the model because of its demonstrated role in visual object processing and in visual retrieval (e.g., Kensinger \& Schacter, 2007; Garoff,
Slotnick, \& Schacter, 2005) and because of recent evidence for important anatomical connections between the amygdala, the hippocampus, and the fusiform gyrus (Smith et al., 2009). ${ }^{1}$ On the basis of anatomical research in nonhumans (Patterson \& Schmidt, 2003; Swanson \& Petrovich, 1998), an anatomical connectivity model was created to specify the anatomically plausible connections between the ROIs (Addis, Moscovitch, \& McAndrews, 2007; McIntosh, 1999) (as in Addis et al., 2007; McIntosh, 1999). Then, a functional model was created for the sleep group and the wake group. Signal change within each ROI was extracted from the ROIs using the MarsBar toolbox (Brett et al., 2002). The average of the signal change across the 4- to 6-sec period poststimulus onset was extracted for successfully recognized negative objects and for missed (forgotten) negative objects. These signal change values were extracted separately for each person and for each region, and a signal change value was computed to reflect the difference in activity for recognized as compared with missed negative objects (i.e., percent signal change to negative object hits-percent signal change to negative object misses). These signal change difference scores were then entered into correlation matrices; separate matrices were created for the sleep and the wake groups, revealing the correlation in signal change among the different ROIs.

A SEM analysis was conducted to examine whether there were group differences in the effective connections among regions during the successful retrieval of negative objects. The functional model (correlation matrix) was entered for each group, and path coefficients were then calculated on the basis of these correlations. Significant differences between the groups were assessed using the stackedmodel approach (McIntosh \& Gonzalez-Lima, 1994). In

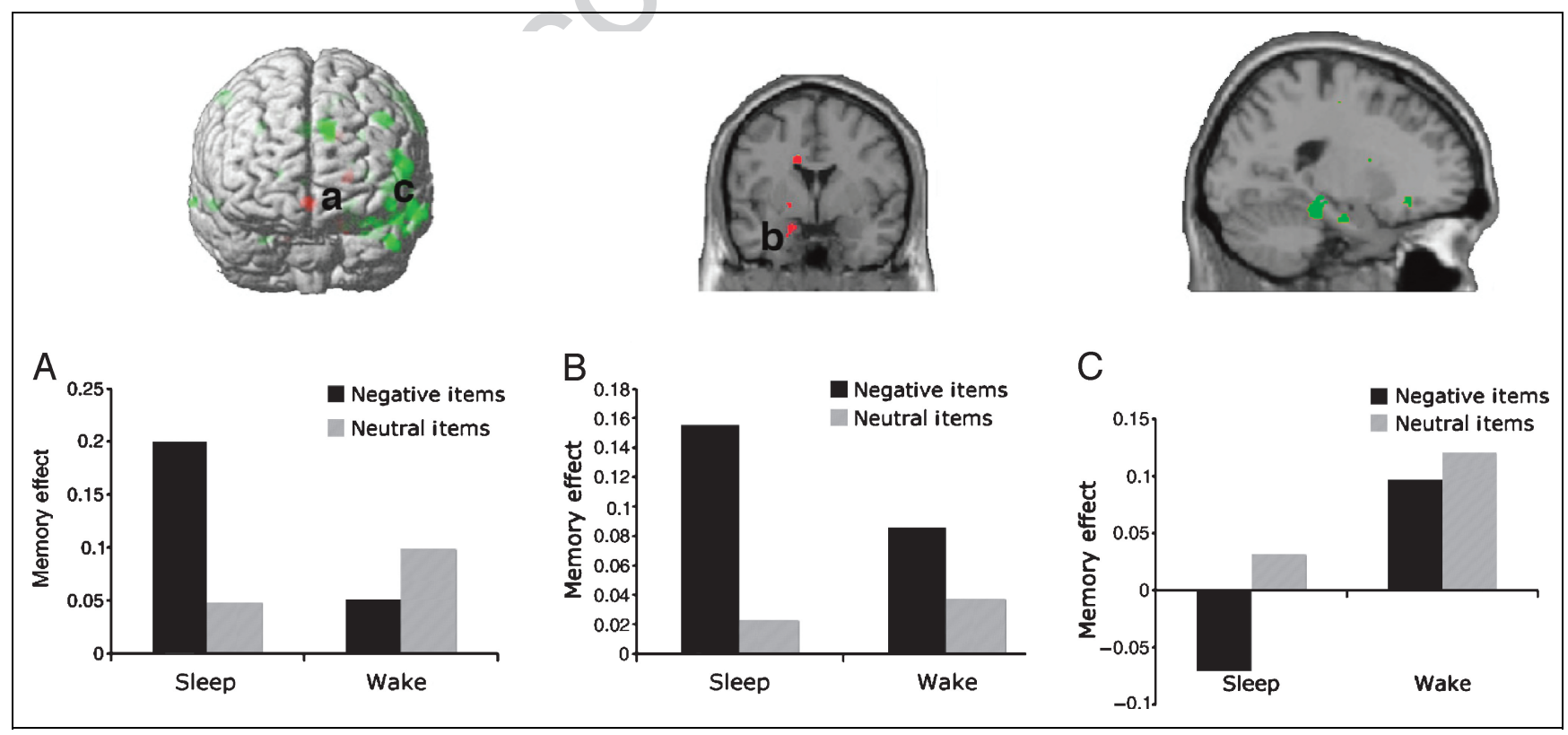

Figure 1. A diffuse network of regions corresponded more strongly with successful retrieval of negative objects after a period of wake (green regions), whereas after a period of sleep, activity was enhanced within a smaller set of limbic regions (in red). Graphs depict the pattern of activity within three of the regions revealed in these group-comparison analyses $(\mathrm{A}=\mathrm{VMPFC} ; \mathrm{B}=$ amygdala; $\mathrm{C}=$ inferior frontal gyrus). 


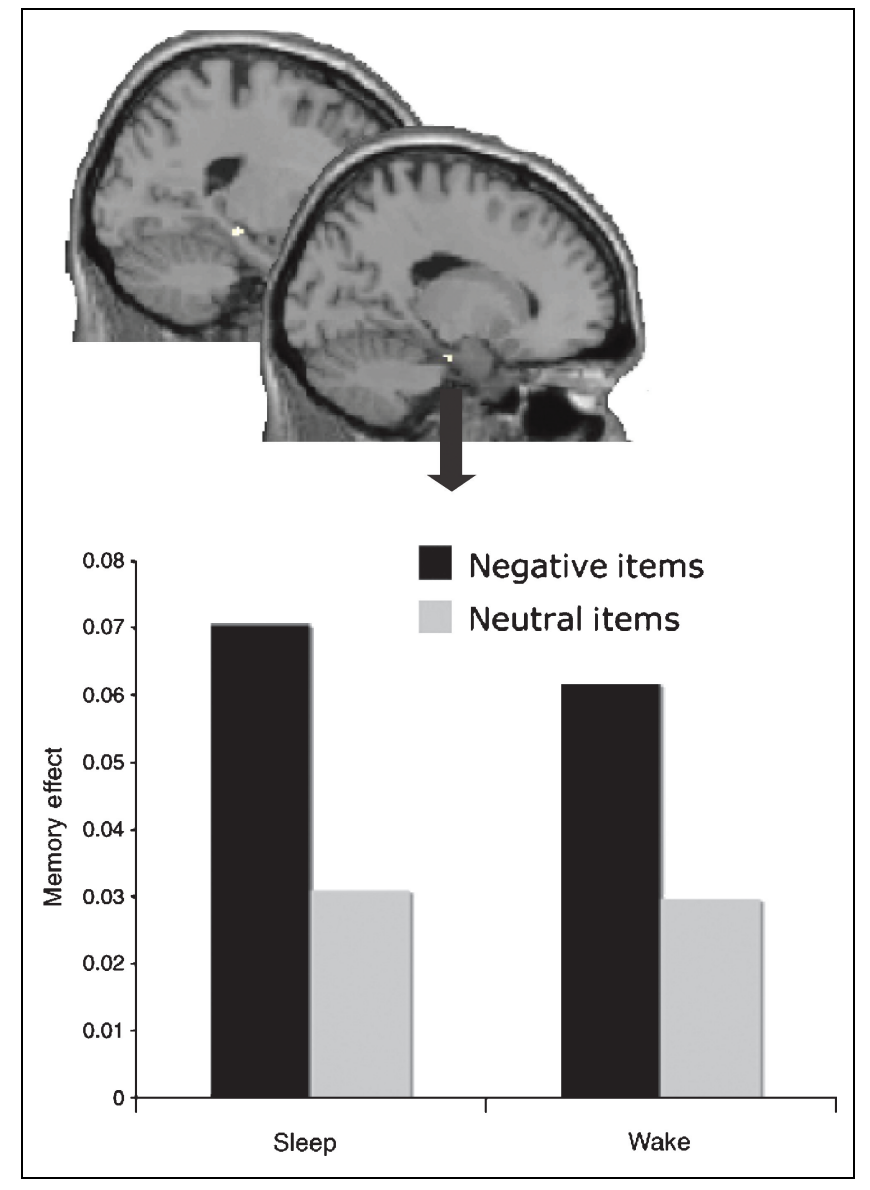

Figure 2. Two regions within the hippocampus (MNI coordinates: -20 , $-26,-10$ and $-16,-13,-22$ ) showed a stronger correspondence to accurate retrieval for the negative items than for the neutral items, but this effect did not interact with the delay group (sleep vs. wake). Graph depicts the strength of the memory effect within the more anterior hippocampal region, as a function of item valence and delay group (sleep vs. wake).

an omnibus test, a null model was first constructed in which the path coefficients from both sleep and wake groups were set to be equal. The fit of this null model was compared with the fit of an alternate model, in which the path coefficients were allowed to differ between the sleep and the wake groups. The goodness-of-fit $\chi^{2}$ values for the two models were compared to determine if one model was a significantly better fit than the other. If the alternate model fits better than the null model, individual connections were allowed to vary in a stepwise manner to determine which connections significantly contributed to the increased fit of the alternate model (i.e., decreased the $p$ value associated with the $\chi^{2}$ difference).

\section{RESULTS}

\section{Behavioral Results}

An ANOVA was conducted on corrected recognition scores (hit rate minus false alarm rate) with scene component (object, background) and scene valence (negative, neutral) as within-subject factors and delay group (sleep, wake) as a between-subject factor. This ANOVA revealed main effects of scene component, $F(1,37)=5.58, p<.05$, partial eta-squared $=.13$, scene valence, $F(1,37)=9.56, p<.01$, partial eta-squared $=.21$, and group, $F(1,37)=8.59, p<$ .01 , partial eta-squared $=.19$. These main effects were qualified by an interaction between scene component and scene valence, $F(1,37)=10.96, p<.001$, partial etasquared $=.75$, and importantly by a three-way interaction among scene component, scene valence, and delay group, $F(1,37)=6.94, p<.05$, partial eta-squared $=.16$. As in Payne, Stickgold, et al. (2008), this three-way interaction demonstrates that sleep preferentially benefits memory for negative objects, $t(37)=4.63, p<.0001$, relative to the other scene components (see Table 1), a finding that cannot easily be reconciled with either a circadian or an interference interpretation of sleep-based memory consolidation (for an in-depth treatment of these issues, see Payne, Stickgold, et al., 2008; Ellenbogen, Payne, \& Stickgold, 2006).

\section{fMRI Group Analysis Results}

Because our behavioral data revealed that sleep had a specific benefit on memory for negative objects relative to wakefulness (consistent with Payne, Stickgold, et al., 2008), we focused our analyses on retrieval of negative objects. Group comparison analyses thus assessed regions showing a stronger correspondence to successful retrieval of negative objects (i.e., stronger activity to hits than to misses) after a period of sleep compared with a period of wake or vice versa. This analysis revealed that, after wakefulness, a diffuse memory network - including widespread activity in the lateral prefrontal and parietal cortices as well as the medialtemporal lobe-corresponded more strongly to successful retrieval of negative items in the wake compared with the sleep group (see upper panel of Table 2 and green regions within Figure 1). The majority of these regions (as denoted by a degree symbol in the rightmost column of Table 2) also showed a three-way interaction between delay group (sleep, wake), memory accuracy (hit, miss), and emotion (negative, neutral). By contrast, a much more refined and restricted network - including the left amygdala, VMPFC, and cingulate gyrus - showed a stronger relation to successful retrieval of negative relative to neutral items after a period of sleep compared with a period of wakefulness (see lower panel of Table 2 and red regions of Figure 1). Finally, a conjunction analysis indicated that two regions within the hippocampus showed a strong correspondence to successful retrieval of negative items regardless of the delay group (i.e., in both sleep and wake conditions, see Figure 2).

\section{fMRI Effective Connectivity Results}

Our next aim was to characterize how regions within an emotional memory network interact with one another after periods of sleep versus wakefulness. We predicted that the amygdala would be a key structure interacting with and 


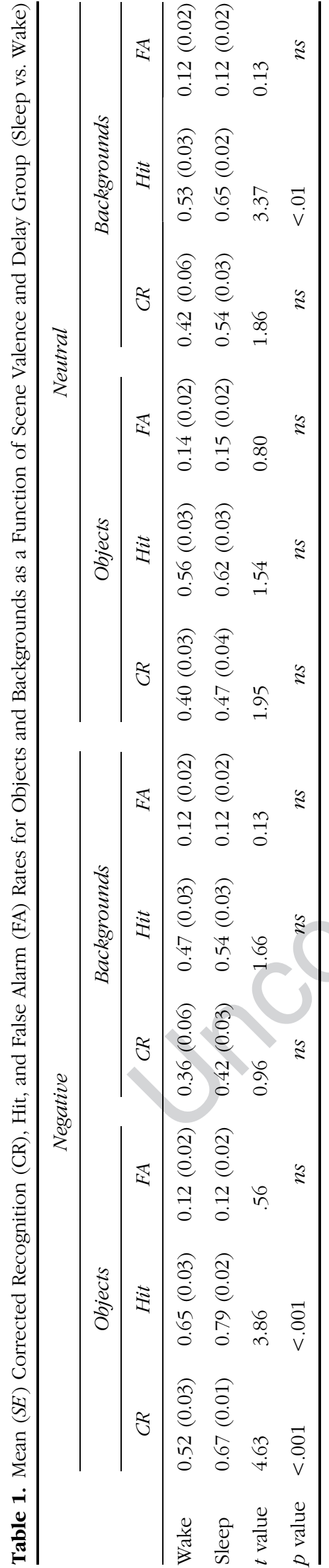

modulating hippocampal function postsleep because coactivation of amygdala and hippocampal regions is critical for boosting consolidation processes and allowing emotional memories to persist over time (McGaugh, 2004; Roozendaal, Nguyen, Power, \& McGaugh, 1999). Moreover, because several neuroimaging studies have revealed a correspondence between VMPFC and fusiform activity and emotional memory retrieval (Buchanan, 2007; Smith, Stephan, Rugg, \& Dolan, 2006; Kensinger \& Schacter, 2005), we included these regions in our model as well (for more information on the definition of these regions, see Methods section).

SEM analysis revealed that for the negative items, the alternate model was a significantly better fit than the null model $(p<.005)$. Subsequent analyses revealed that there were four connections that differed significantly between the sleep group and the wake group: the influence of the amygdala on the hippocampus, the influence of the amygdala on the VMPFC, the influence of the hippocampus on the fusiform gyrus, and the influence of the fusiform gyrus on the amygdala. Of greatest interest here are the differences in amygdala connectivity: After sleep, the amygdala had a stronger positive influence on the hippocampus and on the VMPFC than it did after wake (see Figure 3), suggesting that after sleep the amygdala is more connected with other regions of the emotional memory network. Importantly, the connectivity changes after sleep were specific to the retrieval of the negative items. The null model fit the data for the neutral items, suggesting that during retrieval of neutral items, sleep did not yield significant changes in the connections among the regions included in the model. Thus, sleep does not appear to lead to a ubiquitous change in effective connectivity among emotion processing regions; rather, a night of sleep appears to selectively alter the connectivity during retrieval of negative (but not neutral) events.

\section{DISCUSSION}

Growing behavioral evidence suggests that in addition to stabilizing and enhancing memories, sleep can qualitatively change memories in ways that wakefulness cannot (e.g., Payne et al., 2009; Payne, Stickgold, et al., 2008; Gomez et al., 2006; Wagner et al., 2004). Although these behavioral studies have been taken as evidence that even a single night of sleep is sufficient to lead to an alteration in the way memories are stored and retrieved, neuroimaging methods provide a direct test of this prediction. Using fMRI, we compared the brain regions associated with the retrieval of negatively valenced objects after periods of nocturnal sleep and daytime wakefulness. We predicted that different patterns of neural activity would emerge at retrieval, reflecting the outcome of time spent in these distinct brain states, and also that regions comprising a known emotional memory retrieval network including the amygdala, hippocampus, fusiform gyrus, and VMPFC 
Table 2. Regions That Showed a Stronger Correspondence to Successful Recognition of Negative Items after a Waking Delay as Compared with a Sleeping Delay or Vice Versa ${ }^{\mathrm{b}}$

\begin{tabular}{|c|c|c|c|c|c|}
\hline Region & Gyrus & $\begin{array}{c}\text { Approximate Brodmann's } \\
\text { area (BA) }\end{array}$ & $\begin{array}{c}\text { MNI Coordinates } \\
(x, y, z)\end{array}$ & $\begin{array}{c}\text { TAL Coordinates } \\
(x, y, z)\end{array}$ & $\begin{array}{l}\text { Cluster Size } \\
\text { (No. Voxels) }\end{array}$ \\
\hline \multicolumn{6}{|c|}{ Memory Effect Greater after Waking vs. Sleeping Delay } \\
\hline \multirow[t]{8}{*}{ Frontal lobe } & Inferior frontal gyrus & BA 45 & $-52,20,8$ & $-51,20,6$ & $296^{\mathrm{a}}$ \\
\hline & & $\mathrm{BA} 47^{\mathrm{b}}$ & $-46,38,-10$ & $-46,36,-10$ & 43 \\
\hline & & BA 47 & $-36,24,-20$ & $-36,22,-18$ & 23 \\
\hline & & BA 47 & $-30,20,-20$ & $-30,18,-18$ & 6 \\
\hline & & BA 47 & $-24,30,-8$ & $-24,29,-8$ & 15 \\
\hline & Medial frontal gyrus & BA 6 & $-16,-16,54$ & $-16,-13,50$ & 49 \\
\hline & Middle frontal gyrus & BA 9 & $-40,32,40$ & $-40,32,35$ & 36 \\
\hline & Superior frontal gyrus & BA 9 & $-10,50,30$ & $-10,50,25$ & $132^{\mathrm{a}}$ \\
\hline \multirow[t]{2}{*}{ Cingulate cortex } & Cingulate gyrus & BA 24 & $10,-22,38$ & $10,-20,36$ & 14 \\
\hline & & BA 30 & $-24,-54,14$ & $-24,-52,15$ & $29^{\mathrm{a}}$ \\
\hline \multirow[t]{4}{*}{ Parietal lobe } & Inferior parietal lobule & BA 40 & $-52,-52,52$ & $-51,-48,50$ & $326^{\mathrm{a}}$ \\
\hline & & BA 40 & $50,-38,52$ & $50,-34,50$ & 74 \\
\hline & Postcentral gyrus & BA 2 & $-38,-30,34$ & $-38,-28,33$ & 11 \\
\hline & Precuneus & BA 7 & $-4,-66,34$ & $-4,-62,34$ & 45 \\
\hline Putamen & NA & NA & $-22,4,16$ & $-22,4,15$ & $7^{\mathrm{a}}$ \\
\hline \multirow[t]{13}{*}{ Temporal lobe } & Fusiform gyrus & BA 20 & $-38,-10,-28$ & $-38,-11,-23$ & $44^{\mathrm{a}}$ \\
\hline & Middle temporal gyrus & BA 21 & $-60,0,-12$ & $-60,0,-10$ & $109^{\mathrm{a}}$ \\
\hline & & BA 21 & $56,-14,-10$ & $55,-14,-8$ & 39 \\
\hline & & BA 21 & $70,-20,-8$ & $70,-20,-6$ & 15 \\
\hline & & BA 2 & $-60,-44,-10$ & $-60,-43,-6$ & $64^{\mathrm{a}}$ \\
\hline & & BA 21 & $58,-40,-8$ & $58,-40,-5$ & 26 \\
\hline & & BA 39 & $-42,-64,30$ & $-42,-60,31$ & $264^{\mathrm{a}}$ \\
\hline & Parahippocampal gyrus & BA 35 & $-16,-28,-14$ & $-16,-27,-11$ & 102 \\
\hline & & BA 36 & $-38,-20,-20$ & $-40,-20,-16$ & $72^{\mathrm{a}}$ \\
\hline & & BA 28 & $-18,-14,-22$ & $-18,-14,-18$ & $18^{\mathrm{a}}$ \\
\hline & Superior temporal gyrus & BA 38 & $-46,18,-32$ & $-45,16,-28$ & 58 \\
\hline & & BA 38 & $-50,12,-18$ & $-50,11,-15$ & $63^{\mathrm{a}}$ \\
\hline & & BA 21 & $-56,-24,-2$ & $-55,-23,0$ & $226^{\mathrm{a}}$ \\
\hline \multicolumn{6}{|c|}{ Memory Effect Greater after Sleeping vs. Waking Delay } \\
\hline Amygdala ${ }^{\mathrm{b}}$ & NA & NA & $-18,0,-24$ & $-18,-1,-20$ & $12^{\mathrm{a}}$ \\
\hline Frontal lobe & Medial frontal gyrus & BA 10 & $4,56,-8$ & $4,54,-9$ & $30^{\mathrm{a}}$ \\
\hline \multirow[t]{2}{*}{ Cingulate cortex } & Cingulate gyrus & BA 24 & $-10,-4,32$ & $-10,-2,30$ & $67^{\mathrm{a}}$ \\
\hline & & BA 32 & $-22,36,8$ & $-22,35,5$ & $27^{\mathrm{a}}$ \\
\hline Striatum & NA & NA & $-20,-2,-2$ & $-20,-2,-1$ & 5 \\
\hline Thalamus & NA & NA & $-8,-22,22$ & $-8,-20,21$ & 5 \\
\hline
\end{tabular}

${ }^{a}$ Signifies that at least five of the voxels within this cluster also revealed a three-way interaction between emotion (negative vs. neutral), memory accuracy (remembered, forgotten), and delay group (sleep, wake).

${ }^{\mathrm{b}}$ Regions whose signal change is depicted in Figure 2. 
Figure 3. Group path model of effective connections corresponding with successful retrieval of negative objects after sleep versus wakefulness. Results reveal stronger connections among the vmPFC, amygdala, hippocampus, and fusiform gyrus after a night of sleep than after a day spent awake. Positively weighted paths are represented by solid lines; negatively weighted paths are represented by dotted lines. Thicker arrows indicate stronger connections. Red lines reveal connections that differ significantly between the sleep and the wake groups, whereas black lines show connections that are of comparable strength in the sleep and wake participants. VMPFC $=$ ventromedial $\mathrm{pFC}$ amyg $=$ amygdala; hippo $=$ hippocampus; $\mathrm{PHG}=$ parahippocampal gyrus.

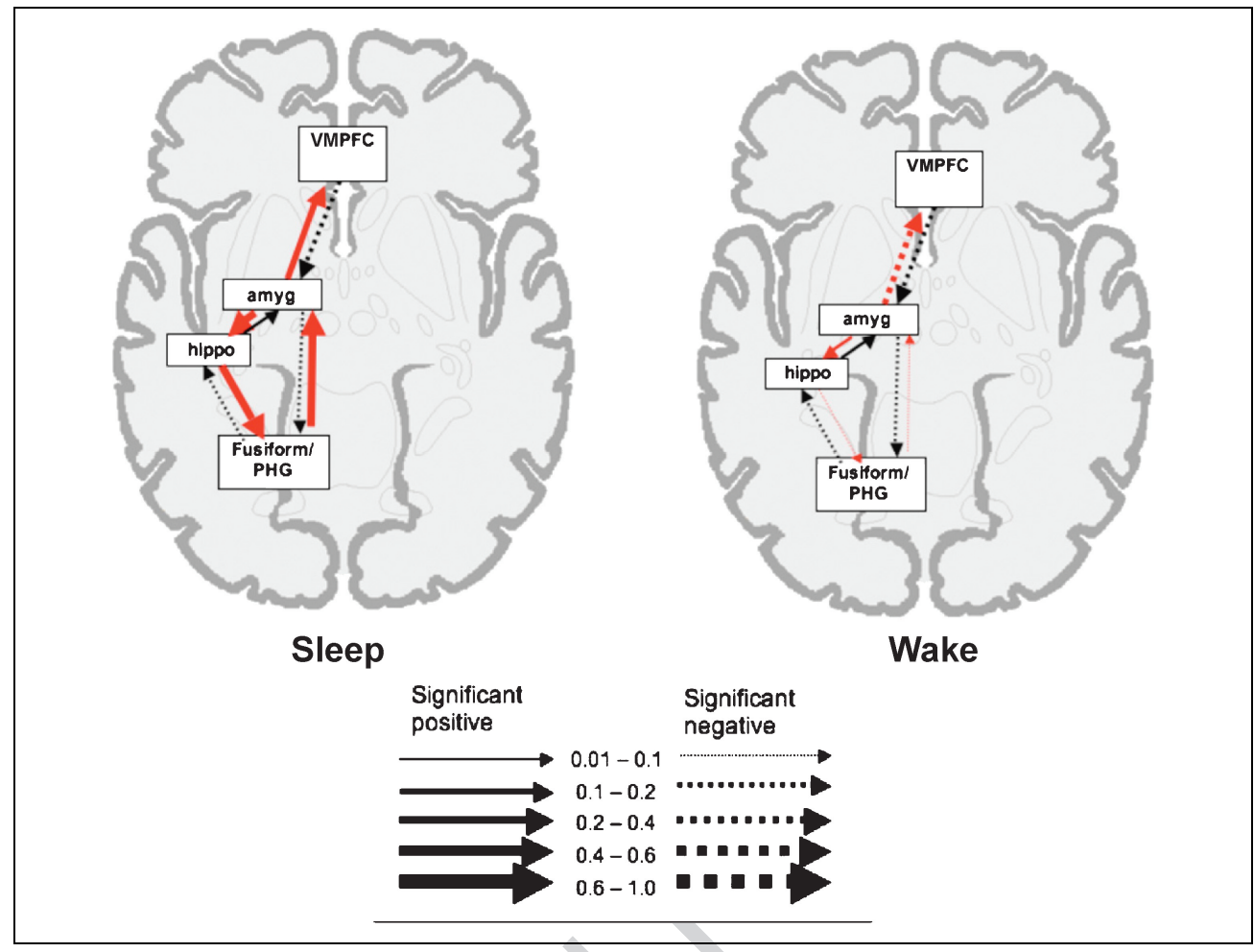

(see reviews by Kensinger, 2009; Buchanan, 2007; LaBar \& Cabeza, 2006) would be more greatly connected after sleep compared with wakefulness.

\section{Activity Analyses Demonstrate That Different Retrieval Networks Are Engaged after Sleep versus Wake}

After a period of wakefulness, successful retrieval of negative objects activated regions that are typically associated with episodic memory retrieval, including widespread activity in the lateral prefrontal, the parietal cortices, and the medial-temporal lobe (Buckner \& Wheeler, 2001; Hanson et al., 1999). Conversely, after a period of sleep, a much more refined and restricted network of regions was activated, including the amygdala, the VMPFC, and the cingulate gyrus. This consolidated pattern of activation is similar to that seen in fMRI investigations of emotional memory retrieval after much longer delays than the 12-hour interval used here (e.g., 1 year in Dolcos, LaBar, \& Cabeza, 2005; 6 months in Sterpenich et al., 2009). One possibility is that a night of sleep can enhance or "speed up" the consolidation process in a way that wakefulness cannot, allowing this refined pattern of activity to emerge after just 12 hours provided that this relatively brief delay contains sleep.

In spite of the substantial differences in activation patterns observed between sleep and wake groups, we did find two regions within the hippocampus that were equally active during successful retrieval of negative objects after sleep and wake, suggesting that the hippocampus is critical for memory retrieval across both types of delay intervals.
The hippocampus appears to be a shared hub used for emotional memory retrieval after a 12-hour delay, regardless of whether it contains sleep or wake. This result fits well with a plethora of findings demonstrating the importance of the hippocampus for memory retrieval after delays of this length or shorter as well as those showing that the hippocampus is often activated in emotional memory tasks similar to the one used here (Buchanan, 2007; Smith et al., 2006; Maratos, Dolan, Morris, Henson, \& Rugg, 2001). The preservation of hippocampal engagement suggests that a night of sleep does not overhaul all of the memory processes recruited for emotional memory retrieval. Rather, the engagement of the hippocampal core of the memory network remains, whereas the number of other nodes of the memory network appears to be reduced after sleep.

\section{Memory Regions Connected to the Amygdala Are Differentially Engaged after Sleep and Wake}

In addition to a reduction in the number of regions engaged after a night of sleep, the effective connectivity analyses revealed that there also was a strengthening in the connectivity among regions within the emotional memory network after a night of sleep. As predicted, the amygdala was more strongly connected to the hippocampus and VMPFC during successful retrieval of emotional objects after sleep than it was after wakefulness. This is the most important finding yielded by the effective connectivity analysis because it suggests that a period of sleep strengthened the modulatory effect of the amygdala on other regions of 
the emotional memory network. Consistent with consolidation theory (McGaugh, 2004), this intensified connectivity between regions likely reflects the strengthening of emotional memories during sleep.

Our results suggest that the emotional memory network is strengthened and refined during sleep, which is in turn reflected in enhanced retrieval of negative relative to neutral objects after sleep relative to wakefulness. These findings are consistent with the claim that consolidation processes serve to mediate emotional memory potentiation over the course of hours to days (LaBar, Gatenby, Gore, LeDoux, \& Phelps, 1998) and with the demonstration that amygdala activity facilitates the induction and expression of plasticity in the hippocampus during sleep, which may be an underlying mechanism of memory consolidation (Datta, Li, \& Auerbach, 2008). Moreover, we provide evidence that a 12-hour delay may be enough time to promote these plastic changes in the neural systems underlying emotional memory, provided it contains a period of sleep.

The VMPFC changes in activity and connectivity observed after sleep are interesting in light of suggestions that this region modulates emotions and facilitates appropriate responding to emotional contexts (Sotres-Bayon, Bush, \& LeDoux, 2004). The medial pFC is thought to activate during retrieval of emotional information to guide appropriate behavioral responses - a task that may be much easier when one has slept. Indeed, a role for this area in sleepbased emotion regulation has been reported (Yoo, Gujar, Hu, Jolesz, \& Walker, 2007). Other evidence suggests that this region, which has reciprocal connections with the medial-temporal lobe and widespread connectivity with disparate neocortical regions, may (like the hippocampus) play a critical role in binding information that is stored in disaggregated cortical areas, allowing for its successful integration at retrieval (Mitchell, Johnson, Raye, \& D'Esposito, 2000).

These findings correspond well with those from the few other neuroimaging studies that have examined emotional memory retrieval processes (for a review, see Buchanan, 2007). For example, previous fMRI studies have identified hippocampal and amygdala activity as necessary components of emotional memory retrieval (Dolcos et al., 2005). Interactions between these regions (Smith et al., 2006) as well as interactions between the amygdala and the fusiform gyrus (Addis, Leclerc, Muscatell, \& Kensinger, 2009; Kensinger \& Schacter, 2008) have been also revealed to be important for memory for negative items in particular. The present data, therefore, suggest that a night of sleep is sufficient to enhance the activity and connectivity of these regions that are tied to successful retrieval of negative information.

The current findings also fit well with a recent study that examined sleep's role in emotional memory retrieval after a more lengthy delay (Sterpenich et al., 2009). These authors compared retrieval of IAPS pictures after nocturnal sleep and after nocturnal sleep deprivation, first after a 3-day delay and again 6 months later. Individuals who were allowed to sleep significantly recruited the medial PFC, precuneus, fusiform gyrus, hippocampus, and amygdala when retrieving emotional memories after the 6-month delay. By contrast, the sleep-deprived participants engaged a distinct network that included increased activity within the amygdala. Although these findings are largely similar to ours, the study of Sterpenich et al. (2009) found additional activation in the precuneus and fusiform gyrus in the sleep group when memories were retrieved after a 6-month delay, whereas we did not find different levels of activation in these regions as a function of sleep (although fusiform connectivity was enhanced in our sleep group).

The differences between the two studies likely derive from the difference in delay intervals (12 hours vs. 6 months) and the use of sleep deprivation as a control condition in the study of Sterpenich et al. (2009), which via marked elevations in stress hormones and nonspecific stress effects can influence all phases of memory processing, including consolidation (Diamond et al., 2006; Payne, Nadel, Britton, \& Jacobs, 2004) and retrieval (de Quervain, Roozendaal, Nitsch, McGaugh, \& Hock, 2000). Elevated stress hormones may also be responsible for differences between the findings of Sterpenich et al. (2009) and those from an earlier study (Sterpenich et al., 2007), which tested the same subjects as the 2009 study but after a shorter (3-day) delay. Sleep-based consolidation effects did not become apparent until after the longer (2009) delay, and we suspect this is because the shorter delay (2007) results were affected by the sleep deprivation manipulation in the wake group. Specifically, Sterpenich et al. (2007) found stronger amygdala activity after wakethe opposite of what is seen in Sterpenich et al. (2009) and in the current study. One possibility is that the stress effects of sleep deprivation intensified amygdala activation during early consolidation processes (for a review, see Diamond et al., 2006; Payne et al., 2004), in which case one would have to wait longer for this to wash out before being able to clearly see the benefit of sleep (as was seen after the longer delay in the 2009 article). Indeed, these lasting effects of stress are one reason we decided to use daytime wakefulness as a comparison group for sleep rather than sleep deprivation as in the Sterpenich et al. (2007, 2009) studies. Although both procedures have strengths and weaknesses, we believe that the two procedures are complementary and together make a strong case for neural reorganization and consolidation during sleep.

In spite of these differences, the current study shares important similarities with Sterpenich et al. (2009), highlighting the importance of limbic and VMPFC regions for successful emotional memory retrieval after a delay that contains sleep. In addition, our results emphasize that many of the limbic changes after long delays in the study of Sterpenich et al. (6 months) can emerge after a single night of sleep, suggesting that the neural plasticity evoked by sleep may be relatively fast acting and long lasting. 
These two neuroimaging studies, along with the mounting behavioral evidence indicating an important role for sleep in emotional memory retrieval (Payne, Stickgold, et al., 2008; Hu et al., 2006; Wagner et al., 2001), suggest that interactions among neural regions involved in memory and emotion are critical for remembering biologically and socially significant information and that sleep plays an essential role in committing this information to long-term memory.

\section{Alternative Explanations for the Present Findings}

At present, there are several major theories offering explanations for how sleep influences memory consolidation. First, it has been suggested that sleep does not confer an active benefit to memory but rather (a) provides a passive and temporary shield against interfering stimulation because sleep is a time of reduced sensory input (Wixted, 2004) or (b) apparent sleep-dependent benefits to memory can be explained by time of day differences in training and testing in the sleep and wake groups. To examine interference and circadian influences on memory, we have conducted three experiments using the current paradigm. We demonstrated that the benefit of sleep on emotional object memory seen here (1) is preserved after a 24-hour delay, but only when sleep directly follows encoding of the emotional scenes (Payne et al., submitted), (2) remains after a delay of several months (see also Wagner et al., 2006), and (3) that emotional objects are better remembered after a 90-min nap than two wake control conditions of equal or shorter length (Payne et al., submitted). This carefully controlled nap study compared a nap condition (90-min nap opportunity) with two interference and circadian control conditions. Participants in the first control condition encoded the images at noon with the nap participants but were tested 90 min earlier than nap participants (i.e., at 5:00 p.m. instead of 6:30 p.m.). Participants in the second control condition arrived 90 min later than the nap participants (i.e., at 1:30 p.m. instead of noon) but were tested at the same time as the nap participants (at 6:30 p.m.). Note that this is a very conservative test of sleep-based memory consolidation because participants in the nap group had a longer retention interval (6.5 hours) than participants in either of the control groups ( 5 hours). Yet participants in the nap group recognized more emotional items than participants in either control group, and performance in the nap group correlated with a specific sleep stage-slow-wave sleep.

Although it remains possible that interference or circadian effects might explain the neural differences observed between the sleep and the wake groups in the current study, it seems unlikely given the above behavioral results and the convergence in regions of activation observed in our study and in the study by Sterpenich et al. (2009). As mentioned, a drawback of the Sterpenich et al. study is the use of a sleep-deprived control group. However, an important benefit of using the sleep deprivation procedure is that it controls for interference and circadian effects. Together, the two sets of findings limit concerns about interference and circadian interpretations (see also Ellenbogen et al., 2006). Nonetheless, we acknowledge that it is impossible to fully rule out circadian differences between wake and sleep groups in our design. Optimal time of day for encoding could differ from the optimal time of day for retrieval, which makes it difficult to control for circadian effects at both time points while also manipulating nocturnal sleep unless the potential confound of sleep deprivation is introduced. We have used nap designs to eliminate this confound in our behavioral studies, and future neuroimaging studies should do the same (although there may be important differences between daytime napping and nocturnal sleep). We believe that the clearest advances will be made by finding corroborating evidence in studies using sleep deprivation, studies using carefully controlled nap designs, and studies using sleeping versus waking delays as we have done here.

A second theory argues that learning results in a net increase in synaptic strength in neural circuits involved in learning that is not energetically sustainable. By this account, the role of sleep is to "downscale" synaptic strength to a baseline level, allowing only the strongest of synapses, and thus memories, to survive (thus making them appear preserved or enhanced by sleep). This position is inconsistent with the current data, however, because it is unclear how it could account for the different neural regions activated after sleep and wake. Moreover, it is unclear how it can reconcile the activation differences observed in our sleep group with those we observed during successful encoding of the same negative objects (Waring \& Kensinger, submitted; that is, superior frontal gyrus, inferior and middle temporal gyrus, fusiform gyrus, and a region of the amygdala centered at different coordinates than those seen here).

A third theory suggests that sleep provides ideal conditions for systems consolidation, which involves communication and coordinated reactivation of the neural patterns between hippocampal and neocortical areas, leading to the gradual integration of memories within neocortical networks (Maquet, 2001; Stickgold, Hobson, Fosse, \& Fosse, 2001; McClelland, McNaughton, \& O’Reilly, 1995). Our results are consistent with this theory and suggest that the beginnings of systems level consolidation can be observed after a 12-hour delay, provided it contains sleep. This finding is in line with several other studies examining the influence of sleep on systems consolidation (Sterpenich et al., 2009; Gais et al., 2007), with one study suggesting that a mere 90 min of sleep is sufficient to trigger this process (Takashima et al., 2006). Together, these neuroimaging studies make a strong argument for the unique neurobiological milieu of sleep as a possible facilitator or even triggering mechanism for systems consolidation and reorganization.

This reorganization of memory traces across macroscopic brain systems or "transfer" as it is sometimes called 
occurs as memories become less dependent on the hippocampus and are increasingly supported by neocortical networks (Squire, 1992). Interestingly, memories may be fundamentally altered as they undergo this type of reorganization, perhaps leading to the qualitative changes seen in the behavioral experiments noted earlier (Payne et al., 2009; Payne, Stickgold, et al., 2008; Dumay \& Gaskell, 2007; Gomez et al., 2006; Wagner et al., 2004). In each of these studies, sleep appears to trigger a flexibility in memory that may be at times more advantageous than a literal representation of experience.

Altogether, we believe that our results are best explained by a consolidation account, where interactions between the amygdala and other regions of the emotional memory network during sleep act to improve memory for negative objects over neutral ones. ${ }^{2}$ It is worth noting that this interpretation of our neuroimaging data fits well with studies of sleep-based memory consolidation stemming from molecular, cellular, and electrophysiological levels of analysis (e.g., Datta et al., 2008; for a review, see Payne, Ellenbogen, et al., 2008; Payne, Stickgold, et al., 2008; Marshall \& Born, 2007; Rasch \& Born, 2007; Stickgold, 2001, 2005; Wilson, 2002; Maquet, 2001).

Being able to remember emotional information and experiences is essential for an adaptive memory system. Our memory likely evolved to help us recall negative objects, such as the appearance of a predator, rather than other, more neutral, occurrences (Nairne, Thompson, \& Pandeirada, 2007). Accordingly, an enhancement of emotional, relative to neutral, stimuli is well established (for a review, see Payne et al., 2004). However, although much is known about emotional memory encoding in humans, the mechanisms supporting the consolidation and retrieval of emotional memories, particularly over long delays, have received less attention. Like other studies, our results provide evidence that activation of and interactions between the hippocampus, amygdala, and $\mathrm{pFC}$ are important for emotional memory retrieval (for a review, see Buchanan et al., 2007). Moreover, they provide evidence that this emotional memory retrieval network is more strongly activated and more strongly connected after a 12 -hour period containing nocturnal sleep than after 12 hours of daytime wakefulness. Critically, sleep appears to refine and intensify connectivity in this emotional memory network, most likely as a function of consolidation processes occurring during the delay interval.

In summary, the present study yielded three major findings that contribute to an understanding of sleep-dependent memory consolidation of emotional information. First, we confirm that a period of nocturnal sleep (relative to daytime wakefulness) preferentially consolidates memory for negative relative to neutral objects (see also Payne, Ellenbogen, et al., 2008; Payne, Stickgold, et al., 2008; Hu et al., 2006; Wagner et al., 2001, 2006). Second, we demonstrate that distinct patterns of brain activation are associated with memory for negative relative to neutral objects depending on whether a 12-hour delay interval contained sleep or wake. This divergence suggests that the qualitative changes seen in memory after nocturnal sleep versus daytime wakefulness in behavioral studies are mirrored by changes in the brain. Third, we demonstrate that interactions between critical affective and mnemonic structures are modulated by whether the postencoding brain state is one of wakefulness or sleep. Together, these findings suggest that sleep profoundly influences the consolidation of emotional memories by modifying the neural architecture used for later retrieval.

\section{Acknowledgments}

The authors thank Donna Addis for her training in effective connectivity analyses, Jon Chamberlain for his assistance with subject running, and Nathan Spreng and Jason Mitchell their for comments on an earlier version of this manuscript.

Reprint requests should be sent to Elizabeth A. Kensinger, Department of Psychology, Boston College, 140 Commonwealth Ave., Chestnut Hill, MA 02467, or via e-mail: elizabeth.kensinger.1@ bc.edu.

\section{Notes}

1. The critical findings from the SEM analyses-that connectivity was increased between the amygdala and the hippocampus and between the amygdala and the VMPFC after sleep-were also revealed in a model that did not include the fusiform gyrus. Here we report the data from the model including the fusiform gyrus because it better captures the set of regions engaged for this type of emotional object processing.

2. To examine whether the differences noted here could reflect greater amygdala engagement in the morning than in the evening, we examined limbic activity across time of day in several studies conducted in our laboratory. We found no evidence that activity varied as a function of circadian time (all $p s>.25$ ). We also found no evidence that the effects of sleep could simply reflect retrieval of stronger memory traces: When we performed a median split of the wake participants on the basis of their recognition performance for the negative items, we found that connections from the vmPFC to the amygdala, from the fusiform to the hippocampus, and from the hippocampus to the fusiform were significantly stronger in the wake participants with the poorest memory. This pattern is opposite of what would be expected if the strengthened connectivity after sleep were due to solely to the strengthened memory trace.

\section{REFERENCES}

Addis, D. R., Leclerc, C. M., Muscatell, K., \& Kensinger, E. A. (2009). There are age-related changes in neural connectivity during the encoding of positive, but not negative, information. Cortex, 46, 425-33.

Addis, D. R., Moscovitch, M., \& McAndrews, M. P. (2007). Consequences of hippocampal damage across the autobiographical memory network. Brain, 130, 2327-2342.

Bartlett, F. C. (1932). Remembering. Cambridge, UK: Cambridge University Press.

Beck, A. T., \& Beamesderfer, A. (1974). Assessment of depression: The depression inventory. Modern Problems of Pharmacopsychiatry, 7, 151-169.

Beck, A. T., Epstein, N., Brown, G., \& Steer, R. A. (1988). An inventory for measuring clinical anxiety: Psychometric 
properties. Journal of Consulting and Clinical Psychology, 56, 893-897.

Brett, M., Anton, J. L., Valabregue, R., \& Poline, J. B. (2002). Region of interest analysis using an SPM toolbox. Paper presented at the 8th International Conference on Functional Mapping of the Human Brain, Sendai, Japan. Available on CD-ROM in Neuroimage, 16.

Buchanan, T. W. (2007). Retrieval of emotional memories. Psychological Bulletin, 133, 761-779.

Buckner, R. L., \& Wheeler, M. E. (2001). The cognitive neuroscience of remembering. Nature Reviews, 2, 624-634.

Dale, A. M. (1999). Optimal experimental design for event-related fMRI. Human Brain Mapping, 8, 109-114.

Datta, S., Li, G., \& Auerbach, S. (2008). Activation of phasic pontine-wave generator in the rat: A mechanism for expression of plasticity-related genes and proteins in the dorsal hippocampus and amygdala. European Journal of Neuroscience, 27, 1876-1892.

de Quervain, D. J., Roozendaal, B., Nitsch, R. M., McGaugh, J. L., \& Hock, C. (2000). Acute cortisone administration impairs retrieval of long-term declarative memory in humans. Nature Neuroscience, 3, 313-314.

Diamond, D. M., Campbell, A. M., Park, C. R., Woodson, J. C., Conrad, C. D., Bachstetter, A. D., et al. (2006). Influence of predator stress on the consolidation versus retrieval of long-term spatial memory and hippocampal spinogenesis. Hippocampus, 16, 571-576.

Dolcos, F., LaBar, K. S., \& Cabeza, R. (2005). Remembering one year later: Role of the amygdala and the medial temporal lobe memory system in retrieving emotional memories. Proceedings of the National Academy of Sciences, U.S.A., 102, 2626-2631.

Dudai, Y. (2004). The neurobiology of consolidations, or, how stable is the engram? Annual Review of Psychology, $55,51-86$

Dumay, N., \& Gaskell, M. G. (2007). Sleep-associated changes in the mental representation of spoken words. Psychological Science, 18, 35-39.

Ellenbogen, J. M., Hu, P. T., Payne, J. D., Titone, D., \& Walker, M. P. (2007). Human relational memory requires time and sleep. Proceedings of the National Academy of Sciences, U.S.A., 104, 7723-7728.

Ellenbogen, J. M., Payne, J. D., \& Stickgold, R. (2006) The role of sleep in declarative memory consolidation: Passive, permissive, active or none? Current Opinion in Neurobiology, 16, 716-722.

Fenn, K. M., Nusbaum, H. C., \& Margoliash, D. (2003). Consolidation during sleep of perceptual learning of spoken language. Nature, 425, 614-616.

Fisher, R. A. (1950). Statistical methods for research workers. London: Oliver and Boyd.

Gais, S., Albouy, G., Boly, M., Dang-Vu, T. T., Darsaud, A., Desseilles, M., et al. (2007). Sleep transforms the cerebral trace of declarative memories. Proceedings of the National Academy of Sciences, U.S.A., 104, 18778-18783.

Gais, S., Lucas, B., \& Born, J. (2006). Sleep after learning aids memory recall. Learning \& Memory (Cold Spring Harbor, N.Y.), 13, 259-262.

Garoff, R. J., Slotnick, S. D., \& Schacter, D. L. (2005). The neural origins of specific and general memory: The role of the fusiform cortex. Neuropsychologia, 43, 847-859.

Gomez, R. L., Bootzin, R. R., \& Nadel, L. (2006). Naps promote abstraction in language-learning infants. Psychological Science, 17, 670-674.

Horne, J. A., \& Ostberg, O. (1976). A self-assessment questionnaire to determine morningness-eveningness in human circadian rhythms. International Journal of Chronobiology, 4, 97-110.
Hu, P., Stylos-Allan, M., \& Walker, M. P. (2006). Sleep facilitates consolidation of emotional declarative memory. Psychological Science, 17, 891-898.

Jenkins, J. G., \& Dallenbach, K. M. (1924). LXXII. Obliviscence during sleep and waking. American Journal of Psychology, 35, 605-612.

Joreskog, K. G., \& Sorbom, D. (1993). LISREL 8: Structural equation modeling with the SIMPLIS command language. Hillsdale, NJ: Earlbaum Associates.

Kensinger, E. A., Garoff-Eaton, R. J., \& Schacter, D. L. (2007). How negative emotion enhances the visual specificity of a memory. Journal of Cognitive Neuroscience, 19, 1872-1887.

Kensinger, E. A., \& Schacter, D. L. (2005). Retrieving accurate and distorted memories: Neuroimaging evidence for effects of emotion. Neuroimage, 27, 167-177.

Kensinger, E. A., \& Schacter, D. L. (2007). Remembering the specific visual details of presented objects: Neuroimaging evidence for effects of emotion. Neuropsychologia, 45, 2951-2962.

Kensinger, E. A., \& Schacter, D. L. (2008). Neural processes supporting young and older adults' emotional memories. Journal of Cognitive Neuroscience, 20, 1161-1173.

LaBar, K. S., Gatenby, J. C., Gore, J. C., LeDoux, J. E., \& Phelps, E. A. (1998). Human amygdala activation during conditioned fear acquisition and extinction: A mixed-trial fMRI study. Neuron, 20, 937-945.

Lazar, N. A., Luna, B., Sweeney, J. A., \& Eddy, W. F. (2002). Combining brains: A survey of methods for statistical pooling of information. Neuroimage, 16, 538-550.

Maquet, P. (2001). The role of sleep in learning and memory. Science, 294, 1048-1052.

Maratos, E. J., Dolan, R. J., Morris, J. S., Henson, R. N., \& Rugg, M. D. (2001). Neural activity associated with episodic memory for emotional context. Neuropsychologia, 39, 910-920.

Marshall, L., \& Born, J. (2007). The contribution of sleep to hippocampus-dependent memory consolidation. Trends in Cognitive Sciences, 11, 442-450.

McClelland, J. L., McNaughton, B. L., \& O’Reilly, R. C. (1995). Why there are complementary learning systems in the hippocampus and neocortex: Insights from the successes and failures of connectionist models of learning and memory. Psychological Review, 102, 419-457.

McDermott, K. B. (1996). The persistence of false memories in list recall. Journal of Memory and Language, 35, 212-230.

McGaugh, J. L. (2000). Memory-A century of consolidation. Science, 287, 248-251.

McGaugh, J. L. (2004). The amygdala modulates the consolidation of memories of emotionally arousing experiences. Annual Review of Neuroscience, 27, 1-28.

McIntosh, A. R. (1999). Mapping cognition to the brain through neural interactions. Memory, 7, 523-548.

McIntosh, A. R., \& Gonzalez-Lima, F. (1994). Network interactions among limbic cortices, basal forebrain, and cerebellum differentiate a tone conditioned as a Pavlovian excitor or inhibitor: Fluorodeoxyglucose mapping and covariance structural modeling. Journal of Neurophysiology, 72, 1717-1733.

Mitchell, K. J., Johnson, M. K., Raye, C. L., \& D'Esposito, M. (2000). fMRI evidence of age-related hippocampal dysfunction in feature binding in working memory. Brain Research, 10, 197-206.

Nairne, J. S., Thompson, S. R., \& Pandeirada, J. N. (2007). Adaptive memory: Survival processing enhances retention. Journal of Experimental Psychology: Learning, Memory, and Cognition, 33, 263-273. 
Patterson, D. W., \& Schmidt, L. A. (2003). Neuroanatomy of the human affective system. Brain and Cognition, 52, 24-26.

Payne, J. D., Ellenbogen, J. M., Walker, M. P., \& Stickgold, R. (2008). The role of sleep in memory consolidation.

In J. H. Byrne (Ed.), Learning and memory: A comprebensive reference. New York: Elsevier.

Payne, J. D., Nadel, L., Britton, W. B., \& Jacobs, W. J. (2004). The biopsychology of trauma and memory. In D. Reisberg \& P. Hertel (Eds.), Memory and emotion (pp. 76-128). New York: Oxford University Press.

Payne, J. D., Schacter, D. L., Propper, R. E., Huang, L. W., Wamsley, E. J., Tucker, M. A., et al. (2009). The role of sleep in false memory formation. Neurobiology of Learning and Memory, 92, 327-334.

Payne, J. D., Stickgold, R., Swanberg, K., \& Kensinger, E. A (2008). Sleep preferentially enhances memory for emotional components of scenes. Psychological Science, 19, 781-788.

Rasch, B., \& Born, J. (2007). Maintaining memories by reactivation. Current Opinion in Neurobiology, 17, 698-703.

Roediger, H. L., \& McDermott, C. M. (2000). Distortions of memory. In E. Tulving \& F. Craik (Eds.), The Oxford handbook of memory (pp. 149-162). New York: Oxford University Press.

Roozendaal, B., Nguyen, B. T., Power, A. E., \& McGaugh, J. L. (1999). Basolateral amygdala noradrenergic influence enables enhancement of memory consolidation induced by hippocampal glucocorticoid receptor activation. Proceedings of the National Academy of Sciences, U.S.A., 96, 11642-11647.

Schacter, D. L. (1996). Memory distortion: How minds, brains and societies reconstruct the past. Cambridge: Harvard University Press.

Smith, A. P., Stephan, K. E., Rugg, M. D., \& Dolan, R. J. (2006). Task and content modulate amygdala-hippocampal connectivity in emotional retrieval. Neuron, 49, 631-638.

Smith, C. D., Lori, N. F., Akbudak, E., Sorar, E., Gultepe, E., Shimony, J. S., et al. (2009). MRI diffusion tensor tracking of a new amygdalo-fusiform and hippocampo-fusiform pathway system in humans. Journal of Magnetic Resonance Imaging, 29, 1248-1261.

Sotres-Bayon, F., Bush, D. E., \& LeDoux, J. E. (2004). Emotional perseveration: An update on prefrontal-amygdala interactions in fear extinction. Learning \& Memory (Cold Spring Harbor, N.Y.), 11, 525-535.
Squire, L. R. (1992). Memory and the hippocampus: A synthesis from findings with rats, monkeys, and humans. Psychological Review, 99, 195-231.

Sterpenich, V., Albouy, G., Darsaud, A., Schmidt, C., Vandewalle, G., Dang Vu, T. T., et al. (2009). Sleep promotes the neural reorganization of remote emotional memory. Journal of Neuroscience, 29, 5143-5152.

Stickgold, R. (2005). Sleep-dependent memory consolidation. Nature, 437, 1272-1278.

Stickgold, R., Hobson, J. A., Fosse, R., \& Fosse, M. (2001). Sleep, learning, and dreams: Off-line memory reprocessing. Science, 294, 1052-1057.

Swanson, L. W., \& Petrovich, G. D. (1998). What is the amygdala? Trends in Neuroscience, 21, 323-331.

Takashima, A., Petersson, K. M., Rutters, F., Tendolkar, I., Jensen, O., Zwarts, M. J., et al. (2006). Declarative memory consolidation in humans: A prospective functional magnetic resonance imaging study. Proceedings of the National Academy of Sciences, U.S.A., 103, 756-761.

Talairach, J., \& Tournoux, P. (1988). Co-planar stereotaxic atlas of the buman brain. New York: Thieme.

Wagner, U., Gais, S., \& Born, J. (2001). Emotional memory formation is enhanced across sleep intervals with high amounts of rapid eye movement sleep. Learning $\varepsilon$ Memory (Cold Spring Harbor, N.Y.), 8, 112-119.

Wagner, U., Gais, S., Haider, H., Verleger, R., \& Born, J. (2004). Sleep inspires insight. Nature, 427, 352-355.

Wagner, U., Hallschmid, M., Rasch, B., \& Born, J. (2006). Brief sleep after learning keeps emotional memories alive for years. Biological Psychiatry, 60, 788-790.

Walker, M. P., \& Stickgold, R. (2006). Sleep, memory, and plasticity. Annual Review of Psychology, 57, 139-166.

Waring, J. D., \& Kensinger, E. A. (2009). Effects of emotional valence and arousal upon memory trade-offs with aging. Psychology and Aging, 24, 412-422.

Wilson, M. A. (2002). Hippocampal memory formation, plasticity, and the role of sleep. Neurobiology of Learning and Memory, 78, 565-569.

Wixted, J. T. (2004). The psychology and neuroscience of forgetting. Annual Review of Psychology, 55, 235-269.

Yoo, S. S., Gujar, N., Hu, P., Jolesz, F. A., \& Walker, M. P. (2007). The human emotional brain without sleep-A prefrontal amygdala disconnect. Current Biology, 17, R877-R878. 


\section{AUTHOR QUERY}

\section{AUTHOR PLEASE ANSWER QUERY}

During the preparation of your manuscript, the question listed below arose. Kindly supply the necessary information.

1. Please check the following references that were cited in the text but not included in the reference list: Payne et al., submitted; Kensinger, 2009; LaBar \& Cabeza, 2006; Hanson et al., 1999; Sterpenich (2009); Sterpenich et al., 2007; Waring \& Kensinger, submitted; Stickgold, 2001; Buchanan et al., 2007.

\section{END OF QUERY}

